\title{
Reversible quantum operations and their application to teleportation
}

\author{
M. A. Nielsen*and Carlton M. Caves \\ Center for Advanced Studies, Department of Physics and Astronomy, \\ University of New Mexico, Albuquerque, NM 87131-1156
}

(July 24, 2018)

\begin{abstract}
Quantum operations provide a general description of the state changes allowed by quantum mechanics. Simple necessary and sufficient conditions for an ideal quantum operation to be reversible by a unitary operation are derived in this paper. These results generalize recent work on reversible measurements by Mabuchi and Zoller [Phys. Rev. Lett. 76, 3108 (1996)]. Quantum teleportation can be understood as a special case of the problem of reversing quantum operations. We characterize completely teleportation schemes of the type proposed by Bennett et al. [Phys. Rev. Lett. 70, 1895 (1993)].

PACS number: 03.65.Bz
\end{abstract}

\section{INTRODUCTION}

Recently Mabuchi and Zoller [1] have shown how a measurement on a quantum system can be reversed under appropriate conditions. In this paper we derive a simple necessary and sufficient condition for an ideal quantum operation to be reversible by a unitary operation. Since all quantum measurements can be described by a set of quantum operations, our result generalizes the scheme of Mabuchi and Zoller. Moreover, it shows how the reversibility of a measurement is connected to the information gained from that measurement.

This paper also examines the teleportation of quantum states, first discussed by Bennett et al. [2]. We show that teleportation can be recast as the problem of reversing a set of quantum operations. The necessary and sufficient condition for unitarily reversing an ideal quantum operation is then applied to give a complete characterization of teleportation schemes of the type proposed by Bennett et al.

The structure of the paper is as follows. In Sec. II, after reviewing the formalism of quantum operations, we define ideal quantum operations and unitarily reversible quantum operations. In Sec. III we review how quantum measurements can be described in terms of a set of quantum operations and show that the scheme of Mabuchi and Zoller is an example of a unitarily reversible ideal quantum measurement. Section IV contains the statement and proof of a general characterization of unitar-

*Electronic address: mnielsen@tangelo.phys.unm.edu ily reversible ideal quantum operations; this characterization generalizes the results of Mabuchi and Zoller. Section V, after reviewing the teleportation scheme described by Bennett et al. [2], formulates the general problem of teleportation and shows how it can be understood as a special case of the problem of reversing a set of quantum operations. Finally, in Sec. VI we use the condition for unitarily reversing an ideal measurement to give a complete characterization of teleportation schemes of the type proposed in [2]. A concluding Sec. VI] summarizes our results.

\section{QUANTUM OPERATIONS}

A simple example of a state change in quantum mechanics is the unitary evolution experienced by a closed quantum system. The final state of the system is related to the initial state by a unitary transformation $U$,

$$
\rho \rightarrow \mathcal{E}(\rho)=U \rho U^{\dagger}
$$

Unitary evolution is not the most general type of state change possible in quantum mechanics. Other state changes, not describable by unitary transformations, arise when a quantum system is coupled to an environment or when a measurement is performed on the system.

How does one describe the most general possible state change in quantum mechanics? The answer to this question is provided by the formalism of "quantum operations." This formalism is described in detail by Kraus [3] and is given a short, but quite informative review by Schumacher in an Appendix [4]. In this formalism there is an input state and an output state, which are connected by a map

$$
\rho \rightarrow \frac{\mathcal{E}(\rho)}{\operatorname{tr}(\mathcal{E}(\rho))} .
$$

This map is determined by a quantum operation $\mathcal{E}$, a linear, trace-decreasing map that preserves positivity. The trace in the denominator is included in order to preserve the trace condition, $\operatorname{tr}(\rho)=1$.

The most general form for $\mathcal{E}$ that is physically reasonable (in addition to being linear and trace-decreasing and preserving positivity, a physically reasonable $\mathcal{E}$ must satisfy an additional property called complete positivity), can be shown to be [3] 


$$
\mathcal{E}(\rho)=\sum_{j} A_{j} \rho A_{j}^{\dagger}
$$

The system operators $A_{j}$, which must satisfy $\sum_{j} A_{j}^{\dagger} A_{j} \leq$ $I$, completely specify the quantum operation. In the particular case of a unitary transformation, there is only one term in the sum, $A_{1}=U$, leaving us with the transformation (2.1).

We now come to the two definitions that delineate the class of reversal problems considered in this paper. We say that a quantum operation $\mathcal{E}$ is ideal if it can be written in the form

$$
\mathcal{E}(\rho)=A \rho A^{\dagger}
$$

for some single operator $A$. The reason for this terminology becomes more apparent in the next section where we discuss ideal quantum measurements.

When we talk about reversing a quantum operation $\mathcal{E}$, we generally do not mean that $\mathcal{E}$ can be reversed for all input states, but rather only that $\mathcal{E}$ can be reversed for a certain class of input states, in particular, for all density operators $\rho$ whose support lies in a subspace $M$ of the total state space $L$. It makes sense to talk about reversing $\mathcal{E}$ on a subspace $M$ only if $\mathcal{E}(\rho) \neq 0$ for all $\rho$ whose support lies in $M$, and we assume this condition henceforth. We say that a quantum operation $\mathcal{E}$ is unitarily reversible on a subspace $M$ if there exists a unitary operator $U$, acting on the total state space $L$, such that

$$
\rho=U \frac{\mathcal{E}(\rho)}{\operatorname{tr}(\mathcal{E}(\rho))} U^{\dagger}
$$

for all $\rho$ whose support lies in $M$.

In this paper our main concern is with unitarily reversing ideal quantum operations. Thus we place two restrictions on the class of reversal problems that we consider: the restriction to reversing ideal operations and the restriction that any reversal be accomplished unitarily.

In principle it is possible to reverse an operation using more general operations than unitary ones. The reversing operation must be deterministic; it turns out that the most general form for a deterministic quantum operation can be obtained by adjoining an ancilla system to the system of interest, allowing the system plus ancilla to interact unitarily, and then discarding the ancilla. Such a dynamics leads to a state change of the form

$$
\rho \rightarrow \operatorname{tr}_{A}\left(V(\rho \otimes \sigma) V^{\dagger}\right) \equiv \mathcal{R}(\rho),
$$

where $\operatorname{tr}_{A}$ denotes tracing out the ancilla, $\sigma$ is the initial state of the ancilla, and $V$ is the unitary operator for the joint dynamics of the system and ancilla.

Such a quantum operation $\mathcal{R}$, called a deterministic or trace-preserving operation, can always [3, 迎 be written in the form

$$
\mathcal{R}(\rho)=\sum_{j} R_{j} \rho R_{j}^{\dagger}
$$

where the system operators $R_{j}$ satisfy the completeness relation $\sum_{j} R_{j}^{\dagger} R_{j}=I$. The completeness relation implies that $\operatorname{tr}(\mathcal{R}(\rho))=\operatorname{tr}(\rho)=1$, thus accounting for the absence of a trace factor to normalize Eq. (2.6). The deterministic operation $\mathcal{R}$ reverses $\mathcal{E}$ on the subspace $M$ if

$$
\rho=\mathcal{R}\left(\frac{\mathcal{E}(\rho)}{\operatorname{tr}(\mathcal{E}(\rho))}\right)
$$

for all $\rho$ whose support lies in $M$.

Even though we restrict the reversal problems considered here to unitarily reversing ideal operations, the restricted problem is still of considerable interest. We show that both the results of Mabuchi and Zoller [1] and the teleportation scheme of Bennett et al. [2] fit within this framework.

\section{QUANTUM MEASUREMENTS}

Standard textbook treatments describe quantum measurements in terms of a complete set of orthogonal projection operators for the system being measured. This formalism, however, does not describe many of the actual measurements that can be performed on a quantum system. The most general type of measurement that can be performed on a quantum system is known as a generalized measurement [3,5].

Generalized measurements can be understood within the framework of quantum operations. The most general type of quantum measurement is described by a set of system operators $A_{i j}$, labeled by two indices, $i$ and $j$, and satisfying the completeness relation

$$
\sum_{i j} A_{i j}^{\dagger} A_{i j}=I .
$$

The first index, $i$, labels the outcome of the measurement. If result $i$ occurs, then the unnormalized state of the system immediately after the measurement is given by

$$
\mathcal{E}_{i}(\rho) \equiv \sum_{j} A_{i j} \rho A_{i j}^{\dagger}
$$

For each measurement result $i$, a different quantum operation $\mathcal{E}_{i}$ describes the corresponding state change.

The probability for result $i$ to occur is

$$
\operatorname{Pr}(i)=\operatorname{tr}\left(\mathcal{E}_{i}(\rho)\right)=\operatorname{tr}\left(\rho \sum_{j} A_{i j}^{\dagger} A_{i j}\right) .
$$

Notice that the measurement probabilities are specified by the positive operators 


$$
E_{i} \equiv \sum_{j} A_{i j}^{\dagger} A_{i j}
$$

The normalization condition, $\sum_{i} \operatorname{Pr}(i)=1$ for all density operators, is equivalent to the completeness condition (3.1), which can be rewritten in terms of the operators $E_{i}$ as

$$
\sum_{i} E_{i}=I
$$

A set of positive operators that satisfy the completeness relation (3.5) is called a positive-operator-valued measure $(\mathrm{POVM})[6]$; the individual operators $E_{i}$ are called POVM elements.

We say a measurement is ideal if for each measurement result $i$, the corresponding quantum operation $\mathcal{E}_{i}$ is ideal; that is, there exist operators $A_{i}$ such that

$$
\mathcal{E}_{i}(\rho)=A_{i} \rho A_{i}^{\dagger} .
$$

The probability that result $i$ occurs is given by

$$
\operatorname{Pr}(i)=\operatorname{tr}\left(\rho A_{i}^{\dagger} A_{i}\right)=\operatorname{tr}\left(\rho E_{i}\right),
$$

where $E_{i}=A_{i}^{\dagger} A_{i}$ is the POVM element for outcome $i$. It can be shown that ideal measurements correspond in a certain sense to doing a perfect readout of the state of the apparatus to which the system is coupled. This is the reason we call such a measurement ideal.

For ideal quantum operations the state change (3.6) corresponding to outcome $i$ can be described in terms of state vectors alone. It becomes

$$
|\psi\rangle \rightarrow \frac{A_{i}|\psi\rangle}{\sqrt{\left\langle\psi\left|A_{i}^{\dagger} A_{i}\right| \psi\right\rangle}},
$$

where the output state vector on the right is only defined up to an arbitrary phase factor. The probability for outcome $i$ takes the form

$$
\operatorname{Pr}(i)=\left\langle\psi\left|A_{i}^{\dagger} A_{i}\right| \psi\right\rangle=\left\langle\psi\left|E_{i}\right| \psi\right\rangle .
$$

This equivalent description in terms of state vectors is often convenient, especially to simplify notation; we use it frequently in the following.

We say that a measurement is unitarily reversible on a subspace $M$ of the total state space $L$ if for each measurement result $i$, the corresponding quantum operation is unitarily reversible; that is, there exists a unitary operator $U_{i}$ such that

$$
U_{i} \frac{\mathcal{E}_{i}(\rho)}{\operatorname{tr}\left(\mathcal{E}_{i}(\rho)\right)} U_{i}^{\dagger}=\rho
$$

for all states $\rho$ whose support lies in $M$. Outcomes that have zero probability on $M$ are irrelevant, because they never occur, and can be discarded; recall that for the other outcomes we assume that $\mathcal{E}_{i}(\rho) \neq 0$ for all $\rho$ whose support lies in $M$.

If the measurement is ideal, then the quantum operations in Eq. (3.10) have the form (3.6) involving a single operator $A_{i}$. For ideal measurements the equivalent definition of a unitarily reversible measurement in terms of state vectors is that for all states $|\psi\rangle$ in the subspace $M$,

$$
U_{i} \frac{A_{i}|\psi\rangle}{\sqrt{\left\langle\psi\left|A_{i}^{\dagger} A_{i}\right| \psi\right\rangle}}=|\psi\rangle,
$$

where equality here is understood to mean equality up to a phase factor. Physically, if the initial state lies in the subspace $M$ and result $i$ occurs, then applying the unitary operator $U_{i}$ to the system returns it to the state it was in before the measurement.

We could define measurements that are only sometimes reversible by requiring that only some of the measurement results have unitarily reversible quantum operations. Although we do not deal explicitly with sometimes reversible measurements in this paper, the results in Sec. IV, since they are derived for individual ideal quantum operations, apply to sometimes reversible measurements.

The scheme proposed by Mabuchi and Zoller [1] is a particular type of unitarily reversible ideal measurement, which can be described as follows. Suppose $a$ and $b$ are annihilation operators for two modes of the electromagnetic field. It is possible in principle to perform an ideal measurement that is described by the following three measurement operators:

$$
\begin{aligned}
A_{1} & =\sqrt{\frac{\Delta}{2}}(a+b), \\
A_{2} & =\sqrt{\frac{\Delta}{2}}(a-b), \\
A_{3} & =e^{-i h \Delta} \sqrt{I-\Delta\left(a^{\dagger} a+b^{\dagger} b\right)} \\
& =I-i h \Delta-\frac{\Delta}{2}\left(a^{\dagger} a+b^{\dagger} b\right) .
\end{aligned}
$$

Here $\Delta$ is an infinitesimal dimensionless time (in the Mabuchi-Zoller scheme, $\Delta$ is an infinitesimal time measured in units of a cavity damping time), and $h$ is a dimensionless Hamiltonian for the modes $a$ and $b$.

The measurement described by the operators (3.12)(3.14) is unitarily reversible on the two-dimensional subspace $M$ spanned by the vectors $\left|2_{a} 0_{b}\right\rangle$ and $\left|0_{a} 2_{b}\right\rangle$. Suppose the system is initially in an arbitrary state in $M$,

$$
|\psi\rangle=\alpha\left|2_{a} 0_{b}\right\rangle+\beta\left|0_{a} 2_{b}\right\rangle .
$$

Since

$$
\begin{aligned}
& A_{1}|\psi\rangle=\sqrt{\Delta}\left(\alpha\left|1_{a} 0_{b}\right\rangle+\beta\left|0_{a} 1_{b}\right\rangle\right) \\
& A_{2}|\psi\rangle=\sqrt{\Delta}\left(\alpha\left|1_{a} 0_{b}\right\rangle-\beta\left|0_{a} 1_{b}\right\rangle\right) \\
& A_{3}|\psi\rangle=e^{-i h \Delta} \sqrt{1-2 \Delta}|\psi\rangle
\end{aligned}
$$


results 1 and 2 each occur with probability $\Delta$, result 3 with probability $1-2 \Delta$, and the post-measurement states for the three results are given by

$$
\begin{array}{ll}
\text { Result 1: } & \alpha\left|1_{a} 0_{b}\right\rangle+\beta\left|0_{a} 1_{b}\right\rangle, \\
\text { Result 2: } & \alpha\left|1_{a} 0_{b}\right\rangle-\beta\left|0_{a} 1_{b}\right\rangle, \\
\text { Result 3: } & e^{-i h \Delta}|\psi\rangle .
\end{array}
$$

It is easy to see that for each measurement result, the original state can be restored by application of an appropriate unitary operation, and Mabuchi and Zoller outline a physically plausible process describing how this unitary operation can be performed in practice when $h=0$. Yet why the measurement can be reversed for initial states of the form (3.15) appears somewhat mysterious in the present example. We now turn to a general result that shows why this is the case.

\section{CHARACTERIZATION OF UNITARILY REVERSIBLE IDEAL QUANTUM OPERATIONS}

In this section we demonstrate that the following conditions are equivalent.

1. The ideal quantum operation $\mathcal{E}(\rho)=A \rho A^{\dagger}$ is unitarily reversible on a subspace $M$ of the total state space $L$.

2. The operator $A^{\dagger} A=E$, when restricted to the subspace $M$, is a positive multiple of the identity operator on $M$; that is,

$$
P_{M} A^{\dagger} A P_{M}=P_{M} E P_{M}=\mu^{2} P_{M}
$$

where $\mu$ is a real constant satisfying $0<\mu \leq 1$ and $P_{M}$ is the projector onto $M$.

3. The quantity $\left\langle\psi\left|A^{\dagger} A\right| \psi\right\rangle=\langle\psi|E| \psi\rangle$ is a positive constant $\mu^{2}$ for all normalized states $|\psi\rangle$ in $M$, where $\mu$ is the real constant of condition 2. If $\mathcal{E}$ represents a measurement result, this means that the probability of occurrence of the result represented by $A$ is the same for all states in $M$. Equivalently, $\operatorname{tr}\left(\rho A^{\dagger} A\right)=\operatorname{tr}(\rho E)=\mu^{2}$ for all density operators whose support lies in $M$.

4. The operator $A$ can be written in the form

$$
A=\mu V P_{M}+A P_{N},
$$

where $V$ is some unitary operator on the whole space $L, \mu$ is the real constant of condition 2 , and $P_{M}$ and $P_{N}$ are the projectors onto the subspaces $M$ and $N$, respectively, where $L=M \oplus N$.
Note first that conditions 2 and 3 are equivalent, 2 being just a restatement of 3 in operator language. In order to prove the other equivalences, we show that 1 implies 2 implies 4 implies 1 . Since 4 implies 1 can be obtained trivially by using $U \equiv V^{\dagger}$ to unitarily reverse the measurement, we only need to prove the other two implications.

Condition 1 implies condition 2. For notational convenience define $B \equiv P_{M} A^{\dagger} A P_{M}$. Considered as an operator on $M, B$ is Hermitian and satisfies $0<B \leq I$; that is, on $M, B$ has a complete set of orthonormal eigenvectors with eigenvalues in the interval $(0,1]$. Suppose that $|1\rangle$ and $|2\rangle$ are two such eigenvectors, with eigenvalues $a_{1}$ and $a_{2}$. Then from condition 1 we have that

$$
U A|1\rangle=\sqrt{a_{1}}|1\rangle \text { and } \quad U A|2\rangle=\sqrt{a_{2}}|2\rangle .
$$

Applying condition 1 to $|\psi\rangle \equiv(|1\rangle+|2\rangle) / \sqrt{2}$ yields

$$
U A|\psi\rangle=\sqrt{\frac{a_{1}+a_{2}}{2}}|\psi\rangle=\frac{\sqrt{a_{1}+a_{2}}}{2}(|1\rangle+|2\rangle),
$$

but since $U A$ is linear, we also have that

$$
\begin{aligned}
U A|\psi\rangle & =\frac{1}{\sqrt{2}}(U A|1\rangle+U A|2\rangle) \\
& =\frac{1}{\sqrt{2}}\left(\sqrt{a_{1}}|1\rangle+\sqrt{a_{2}}|2\rangle\right) .
\end{aligned}
$$

Comparing (4.4) and (4.5) tells us that $a_{1}=a_{2}$ and thus that all the eigenvalues of $B$, considered as an operator on $M$, have the same value $\mu^{2}$. It follows that $B=\mu^{2} P_{M}$.

Condition 2 implies condition 4 . Again define

$$
B \equiv P_{M} A^{\dagger} A P_{M}=\mu^{2} P_{M}
$$

From $P_{M}+P_{N}=I$ we obtain the identity

$$
A=A P_{M}+A P_{N}
$$

The polar decomposition theorem [see Eq. (3.74) of [6] implies that there exists a unitary operator $V$ on $L$ such that

$$
A P_{M}=V \sqrt{P_{M} A^{\dagger} A P_{M}}=V \sqrt{B},
$$

and from $(4.6), 4.7)$, and $(4.8)$, we see that

$$
A=\mu V P_{M}+A P_{N} .
$$

This completes the proof.

It is easy to check that the scheme of Mabuchi and Zoller is an instance of the general result. As we have already noted, for all states in the subspace $M$, results 1 and 2 each occur with probability $\Delta$ and result 3 with probability $1-2 \Delta$. The equivalence of conditions 1 and 3 then implies that the state change for each outcome can be reversed by some unitary operator. 
Condition 4 makes clear formally why an ideal operation described by $A$ can be unitarily reversed on $M$ : when acting on states in $M, A$ acts like the unitary operator $V$, except for rescaling by the real constant $\mu$, which accounts for the probability of obtaining the result corresponding to $A$.

The physical meaning of condition 3 is clear for the set of ideal quantum operations that describe an ideal measurement: an ideal measurement is reversible if and only if no information about the identity of the prior state is obtained from the measurement; more precisely, no inference about the prior state in $M$ can be made, since each state is equally likely, given any result $i$. The necessity of condition 3 for reversing a measurement is obvious: if one could obtain information about the prior state and then restore the prior state, then by repeating the measurement and restoration many times, one could obtain enough information to distinguish nonorthogonal states reliably. The necessity of condition 3 for reversing a single quantum operation, though plausible on the same grounds, is not obvious. Moreover, the important feature of our result is not the necessity, but rather the sufficiency of condition 3 for unitarily reversing an ideal quantum operation.

In view of these remarks it should not be surprising that we can extend the result that condition 1 implies conditions 2 and 3 to apply to deterministic reversal of a general quantum operation, not just unitary reversal of an ideal quantum operation. Besides being of interest in its own right, this extension is used later to show that a necessary condition for teleportation is that the teleportation process obtain no information about the state to be teleported.

Suppose that $\mathcal{E}$ is a general quantum operation, specified by operators $A_{j}$ as in Eq. (2.3), and that $\mathcal{E}$ can be reversed by the deterministic operation $\mathcal{R}$ on a subspace $M$ of the total state space $L$; that is, Eq. (2.8) holds for all density operators $\rho$ whose support lies in $M$. The operator

$$
B \equiv P_{M}\left(\sum_{j} A_{j}^{\dagger} A_{j}\right) P_{M},
$$

considered as an operator on $M$, is Hermitian and satisfies $0<B \leq I$, so $B$ has a complete set of orthonormal eigenvectors on $M$, with eigenvalues in the interval $(0,1]$. Notice that $\operatorname{tr}(\mathcal{E}(\rho))=\operatorname{tr}(\rho B)$ for all density operators $\rho$ whose support lies in $M$.

Suppose that $|1\rangle$ and $|2\rangle$ are any two orthonormal eigenvectors of $B$ in $M$, with eigenvalues $a_{1}$ and $a_{2}$. Define $\rho_{1} \equiv|1\rangle\left\langle 1\left|, \rho_{2} \equiv\right| 2\right\rangle\langle 2|$, and $\rho^{\prime} \equiv \frac{1}{2}\left(\rho_{1}+\rho_{2}\right)$; notice that the support of each of these density operators lies in $M$. Condition (2.8) implies that

$$
\begin{aligned}
& \mathcal{R}\left(\mathcal{E}\left(\rho_{1}\right)\right)=a_{1} \rho_{1}, \\
& \mathcal{R}\left(\mathcal{E}\left(\rho_{2}\right)\right)=a_{2} \rho_{2},
\end{aligned}
$$

$$
\mathcal{R}\left(\mathcal{E}\left(\rho^{\prime}\right)\right)=\frac{1}{2}\left(a_{1}+a_{2}\right) \frac{1}{2}\left(\rho_{1}+\rho_{2}\right),
$$

but from the linearity of $\mathcal{R}$ and $\mathcal{E}$, we also have that

$$
\begin{aligned}
\mathcal{R}\left(\mathcal{E}\left(\rho^{\prime}\right)\right) & =\frac{1}{2}\left[\mathcal{R}\left(\mathcal{E}\left(\rho_{1}\right)\right)+\mathcal{R}\left(\mathcal{E}\left(\rho_{2}\right)\right)\right] \\
& =\frac{1}{2}\left(a_{1} \rho_{1}+a_{2} \rho_{2}\right) .
\end{aligned}
$$

Comparing Eqs. (4.13) and (4.14), we see that $a_{1}=a_{2}$. Thus all the eigenvalues of $B$, considered as an operator on $M$, have the same value $\mu^{2}$, where $0<\mu \leq 1$; that is,

$$
B=P_{M}\left(\sum_{j} A_{j}^{\dagger} A_{j}\right) P_{M}=\mu^{2} P_{M} .
$$

Equivalently, we see that $\operatorname{tr}(\mathcal{E}(\rho))$ is a positive constant, $\mu^{2}$, for all density operators $\rho$ whose support lies in $M$. If $\mathcal{E}$ represents a measurement result, this means that the probability of the result represented by $\mathcal{E}$ is the same for all states whose support lies in $M$.

Thus we see that a necessary condition for reversing a general quantum measurement is that no information about the identity of the prior state be obtained from the measurement. It is clear that this is not also a sufficient condition, since it is easy to construct nonideal quantum operations for which $\operatorname{tr}(\mathcal{E}(\rho))$ is the same for all states whose support lies in a subspace $M$, but which take all states in $M$ to the same final state.

\section{TELEPORTATION AND REVERSIBLE QUANTUM OPERATIONS}

We begin this section with a brief outline of the teleportation scheme described in Bennett et al. [2]. This scheme involves a sender, Alice, and a receiver, Bob. Alice is in possession of two two-level systems, the input system, labeled 1, and another system, labeled 2. Bob is in possession of a third two-level system, labeled 3 . We use $|\uparrow\rangle$ and $|\downarrow\rangle$ to denote an orthonormal set of basis states for each two-level system. It should be noted that Bennett et al. extended this scheme to $n$-level systems.

Initially the composite system is prepared in a state with density operator $\rho \otimes \sigma$, where $\rho$ is an unknown state of the input system 1 , and $\sigma$ is a maximally entangled pure state of systems 2 and 3 ,

$$
\sigma=\frac{1}{2}(|\uparrow \downarrow\rangle+|\downarrow \uparrow\rangle)(\langle\uparrow \downarrow|+\langle\downarrow \uparrow|)
$$

Alice's goal is to "teleport" the input state $\rho$ to the target system, Bob's system 3. This is done as follows. Alice performs a measurement on systems 1 and 2 in the Bell operator basis [7], which consists of four entangled states for systems 1 and 2 , 


$$
\begin{aligned}
& \left|\psi^{ \pm}\right\rangle=\frac{1}{\sqrt{2}}(|\uparrow \downarrow\rangle \pm|\downarrow \uparrow\rangle), \\
& \left|\phi^{ \pm}\right\rangle=\frac{1}{\sqrt{2}}(|\uparrow \uparrow\rangle \pm|\downarrow \downarrow\rangle) .
\end{aligned}
$$

Alice sends the result of this measurement, which we denote by $i=1,2,3$ or 4 , to Bob. It was shown in [2] that there exist unitary operators $U_{i}$, acting only on the target system 3 , which belongs to Bob, such that if Bob performs the unitary operation $U_{i}$ corresponding to measurement result $i$, then the final state of Bob's system is the input state $\rho$. Alice has "teleported" the state $\rho$ to Bob, with the help of the two bits of classical information necessary to tell Bob the result $i$ of her measurement.

We devote the remainder of this section to formulating the problem of teleportation generally; in particular, we show how teleportation can be understood in terms of reversing quantum operations. Suppose Alice has possession of an input system, which we label 1, in an unknown input state $\tilde{\rho}^{1}$. To avoid confusion here and throughout the remainder of this paper, we use a superscript to denote the appropriate state space for a vector or an operator; the reason for the tilde becomes clear shortly. Alice might also have access to another system, which we label 2. Bob has access to the target system, which we label 3. Systems 2 and 3 are assumed to be prepared initially in some standard state $\sigma^{23}$, which is assumed to be uncorrelated with $\tilde{\rho}^{1}$; that is, the initial state of the composite system consisting of 1,2 , and 3 is

$$
\tilde{\rho}^{1} \otimes \sigma^{23} \text {. }
$$

The case where Bob has access to an additional system, labeled 4 , is discussed briefly later in this section.

We assume that systems 1 and 3 are identical and thus have the same state space. This means that there is a one-to-one linear map from the state space of 3 onto the state space of 1 . Though this map is not unique, we choose a particular one, thereby setting up a one-to-one correspondence between vectors in the state space of 3 and vectors in the state space of 1 . We denote this oneto-one correspondence by

$$
\left|\psi^{3}\right\rangle \leftrightarrow\left|\tilde{\psi}^{1}\right\rangle .
$$

The one-to-one correspondence between vectors induces a one-to-one correspondence between operators on 3 and operators on 1 , which we denote by $A^{3} \leftrightarrow \tilde{A}^{1}$. This correspondence is given by linearly extending the map $\left|\psi^{3}\right\rangle\left\langle\phi^{3}|\leftrightarrow| \tilde{\psi}^{1}\right\rangle\left\langle\tilde{\phi}^{1}\right|$ to all operators on systems 3 and 1 . In particular, for each state $\tilde{\rho}^{1}$ of the input system, there is a unique counterpart state $\rho^{3}$ of the target system.

The choice of a correspondence between the state spaces of 1 and 3 is physically motivated: the correspondence defines what it means to transport a system unchanged from the location of system 1 to the location of system 3. Different procedures for performing this transportation lead to different correspondences. For example, suppose we wish to teleport the state of a spin- $\frac{1}{2}$ particle from Albuquerque to Santa Barbara. To say what it means to teleport the state requires a correspondence between the state spaces in Albuquerque and Santa Barbara. We could set up the correspondence by agreeing that the $z$ axis in each location lies along the local acceleration of gravity and the the $x$ axis along the local magnetic north or by adopting arbitrary orthogonal axes in the two locations. Ordinarily we assume implicitly such a correspondence, as is done in the original paper on teleportation, and write $\tilde{\rho}^{1}=\rho^{3}=\rho$.

The correspondence can be extended to a one-to-one correspondence between the joint state space of 2 and 3 and the joint state space of 1 and 2. If $\left|b^{2}\right\rangle\left|c^{3}\right\rangle$ is a product basis for the joint state space of 2 and 3, this one-to-one correspondence is given by

$$
\left|\psi^{23}\right\rangle=\sum_{b, c} \alpha_{b c}\left|b^{2}\right\rangle\left|c^{3}\right\rangle \leftrightarrow \sum_{b, c} \alpha_{b c}\left|\tilde{c}^{1}\right\rangle\left|b^{2}\right\rangle=\left|\tilde{\psi}^{12}\right\rangle .
$$

This correspondence induces a one-to-one correspondence between operators on the joint state space of 2 and 3 and operators on the joint state space of 1 and 2 .

The correspondence can be extended further to a oneto-one linear map from the state space of the composite system 1,2 , and 3 onto itself:

$$
\left|\psi^{123}\right\rangle \leftrightarrow\left|\tilde{\psi}^{123}\right\rangle=U_{13}\left|\psi^{123}\right\rangle .
$$

This map is accomplished by a unitary operator $U_{13}$, which acts on product states according to

$$
U_{13}\left|\tilde{a}^{1}\right\rangle\left|b^{2}\right\rangle\left|c^{3}\right\rangle=\left|\tilde{c}^{1}\right\rangle\left|b^{2}\right\rangle\left|a^{3}\right\rangle
$$

and thus is called the "swap" operator because it swaps the states of systems 1 and 3 , while leaving system 2 alone. The swap operator clearly satisfies $\left(U_{13}\right)^{2}=I^{123}$, that is, $U_{13}^{\dagger}=U_{13}$. When extended to operators on the composite system, the correspondence becomes

$$
A^{123} \leftrightarrow \tilde{A}^{123}=U_{13} A^{123} U_{13}^{\dagger} .
$$

Suppose now that Alice performs a measurement on systems 1 and 2 . This measurement is described by operators $\tilde{A}_{i j}^{12} \otimes I^{3}$, where the operators $\tilde{A}_{i j}^{12}$ are operators on the joint system consisting of 1 and $2, i$ as usual labeling the result of the measurement. If the measurement has outcome $i$, then the unnormalized state of the target system 3 after the measurement is given by

$$
\hat{\rho}_{i}^{3}=\operatorname{tr}_{12}\left(\sum_{j}\left(\tilde{A}_{i j}^{12} \otimes I^{3}\right)\left(\tilde{\rho}^{1} \otimes \sigma^{23}\right)\left[\left(\tilde{A}_{i j}^{12}\right)^{\dagger} \otimes I^{3}\right]\right) .
$$

where the caret denotes an unnormalized state.

We now show that $\hat{\rho}_{i}^{3}$ is related to $\rho^{3}$ by a quantum operation, which we denote $\mathcal{E}_{i}$. We first notice that 


$$
\tilde{\rho}^{1} \otimes \sigma^{23}=U_{13}\left(\tilde{\sigma}^{12} \otimes \rho^{3}\right) U_{13}^{\dagger},
$$

where $\tilde{\sigma}^{12}$ is the counterpart of $\sigma^{23}$. Substituting this into (5.10) gives

$$
\begin{aligned}
\hat{\rho}_{i}^{3}=\operatorname{tr}_{12}( & \sum_{j}\left(\tilde{A}_{i j}^{12} \otimes I^{3}\right) \\
& \left.\times\left[U_{13}\left(\tilde{\sigma}^{12} \otimes \rho^{3}\right) U_{13}^{\dagger}\right]\left[\left(\tilde{A}_{i j}^{12}\right)^{\dagger} \otimes I^{3}\right]\right) .
\end{aligned}
$$

The form of this equation allows us to think of $\hat{\rho}_{i}^{3}$ as arising from the following process. The composite system begins in the state $\tilde{\sigma}^{12} \otimes \rho^{3}$, in which the joint system 1 and 2 is in the state $\tilde{\sigma}^{12}$ and system 3 is in the state $\rho^{3}$. After the composite system evolves under the unitary swap operator, a measurement is performed on the joint system 1 and 2 , and then the joint system 1 and 2 is discarded. This process being a measurement on system 3 , it is not surprising that the state change from $\rho^{3}$ to $\hat{\rho}_{i}^{3}$ is described by a quantum operation. We now show explicitly how to construct the quantum operation $\mathcal{E}_{i}$. This having been done, the problem of teleportation is for Bob to reverse the quantum operation $\mathcal{E}_{i}$. If the reversal can be done, then Bob can recover the state $\rho^{3}$ from the output state $\hat{\rho}_{i}^{3}=\mathcal{E}_{i}\left(\rho^{3}\right)$ of system 3 .

We write

$$
\tilde{\sigma}^{12}=\sum_{k} p_{k}\left|\tilde{s}_{k}^{12}\right\rangle\left\langle\tilde{s}_{k}^{12}\right|
$$

where the vectors $\left|\tilde{s}_{k}^{12}\right\rangle$ make up the complete orthonormal set of eigenvectors of $\tilde{\sigma}^{12}$ in the joint space of 1 and 2 . Furthermore, we let $\tilde{\Pi}_{l}^{12}=\left|\tilde{P}_{l}^{12}\right\rangle\left\langle\tilde{P}_{l}^{12}\right|$ be any complete set of orthogonal one-dimensional projectors for the joint system 1 and 2. Performing the partial trace of Eq. (5.12) in the basis $\left|\tilde{P}_{l}^{12}\right\rangle$ yields

$$
\begin{aligned}
\hat{\rho}_{i}^{3}=\sum_{j k l} & \left(\sqrt{p_{k}}\left\langle\tilde{P}_{l}^{12}\left|\left(\tilde{A}_{i j}^{12} \otimes I^{3}\right) U_{13}\right| \tilde{s}_{k}^{12}\right\rangle\right) \rho^{3} \\
& \times\left(\sqrt{p_{k}}\left\langle\tilde{s}_{k}^{12}\left|U_{13}^{\dagger}\left[\left(\tilde{A}_{i j}^{12}\right)^{\dagger} \otimes I^{3}\right]\right| \tilde{P}_{l}^{12}\right\rangle\right) .
\end{aligned}
$$

Using the single index $m$ to denote the triple $(j, k, l)$ and defining the system 3 operators

$$
\begin{aligned}
B_{i m}^{3} & \equiv \sqrt{p_{k}}\left\langle\tilde{P}_{l}^{12}\left|\left(\tilde{A}_{i j}^{12} \otimes I^{3}\right) U_{13}\right| \tilde{s}_{k}^{12}\right\rangle \\
& =\sqrt{p_{k}}\left\langle\tilde{P}_{l}^{12}\left|U_{13}\left(I^{1} \otimes A_{i j}^{23}\right)\right| \tilde{s}_{k}^{12}\right\rangle,
\end{aligned}
$$

we can write the output state of system 3 as

$$
\hat{\rho}_{i}^{3}=\sum_{m} B_{i m}^{3} \rho^{3}\left(B_{i m}^{3}\right)^{\dagger} \equiv \mathcal{E}_{i}\left(\rho^{3}\right) .
$$

As we set out to show, $\hat{\rho}_{i}^{3}$ is related to $\rho^{3}$ by a quantum operation $\mathcal{E}_{i}$.
Notice that because of the sums introduced by the partial trace and the orthogonal decomposition of $\tilde{\sigma}^{12}$, the quantum operations $\mathcal{E}_{i}$ generally are not ideal even if the measurement on 1 and 2 is ideal. In the next section we explore a case where the quantum operations $\mathcal{E}_{i}$ are ideal.

For Bob to perform teleportation, he must now perform a deterministic quantum operation $\mathcal{R}_{i}$ on system 3 such that

$$
\mathcal{R}_{i}\left(\frac{\mathcal{E}_{i}\left(\rho^{3}\right)}{\operatorname{tr}\left(\mathcal{E}_{i}\left(\rho^{3}\right)\right)}\right)=\rho^{3} .
$$

We have shown that the problem of understanding teleportation can be reduced to the problem of understanding how to reverse quantum operations. Given the work that has been done on reversing deterministic quantum operations that arise from decoherence, this would seem to be a useful insight (see [1, 814 for a sample of this work).

In this paper we are mainly interested in the case where Bob does the reversal using unitary quantum operations. Notice that if Bob had access to an additional system, 4, then he could perform nonunitary, but still deterministic quantum operations in order to restore the original input state. Using the earlier result that a necessary condition for reversing a general quantum operation is that the operation yield no information about the input state, we see that a necessary condition for teleportation is that Alice gain from her measurement no information about the state of the input system. In this paper we concentrate on unitary reversal of ideal quantum operations, so beyond this remark, we do not consider the case where Bob has access to an extra system.

Ideal quantum operations arise naturally in the teleportation scheme considered by Bennett et al. and might also arise in other schemes. In this case we seek unitary operators $U_{i}$ such that $\mathcal{E}_{i}$ is unitarily reversible by $U_{i}$,

$$
U_{i} \frac{\mathcal{E}_{i}\left(\rho^{3}\right)}{\operatorname{tr}\left(\mathcal{E}_{i}\left(\rho^{3}\right)\right)} U_{i}^{\dagger}=\rho^{3} .
$$

This is precisely the condition that is required to achieve teleportation!

\section{CHARACTERIZATION OF TELEPORTATION SCHEMES}

In this section we consider teleportation schemes of the type introduced by Bennett et al. [2]. Suppose we have a composite system made up of three parts, each with the same $d$-dimensional state space $H$, so the state space of the composite system is $H^{1} \otimes H^{2} \otimes H^{3}$. Alice has possession of systems 1 and 2, and Bob has possession of system 3. In the scheme of Bennett et al. outlined earlier, $H$ is a two-dimensional state space. 
The composite system is prepared in the state

$$
\tilde{\rho}^{1} \otimes \sigma^{23}
$$

where $\tilde{\rho}^{1}$ is any state (pure or mixed) of system 1 and

$$
\sigma^{23}=\left|s^{23}\right\rangle\left\langle s^{23}\right|
$$

is a pure state of the joint system made up of systems 2 and 3 . In the case considered by Bennett et al., $\sigma^{23}$ is a maximally entangled pure state of systems 2 and 3 .

Alice performs a joint measurement on systems 1 and 2. We assume that this measurement is an ideal measurement described by measurement operators

$$
\sqrt{\gamma_{i}} \tilde{\Pi}_{i}^{12} \otimes I^{3},
$$

where the operators

$$
\tilde{\Pi}_{i}^{12}=\left|\tilde{P}_{i}^{12}\right\rangle\left\langle\tilde{P}_{i}^{12}\right|
$$

are one-dimensional projectors onto the joint system made up of 1 and 2 and the $\gamma_{i}$ are real constants satisfying $0<\gamma_{i} \leq 1$ 15. The measurement operators satisfy a completeness relation, which in this case becomes

$$
\sum_{i} \gamma_{i} \tilde{\Pi}_{i}^{12}=\sum_{i} \gamma_{i}\left|\tilde{P}_{i}^{12}\right\rangle\left\langle\tilde{P}_{i}^{12}\right|=I^{12},
$$

but they need not be orthogonal - that is, the vectors $\left|\tilde{P}_{i}^{12}\right\rangle$ can be an overcomplete set of nonorthogonal vectors. If the projection operators are orthogonal, then all the constants $\gamma_{i}=1$. As remarked earlier, in the scheme of Bennett et al., the operators describing the measurement are projectors onto an orthonormal Bell basis for the joint system 1 and 2 .

As in the preceding section, we can write

$$
\tilde{\rho}^{1} \otimes \sigma^{23}=U_{13}\left(\tilde{\sigma}^{12} \otimes \rho^{3}\right) U_{13}^{\dagger},
$$

where $\tilde{\sigma}^{12}=\left|\tilde{s}^{12}\right\rangle\left\langle\tilde{s}^{12}\right|$, the counterpart of $\sigma^{23}$, is a pure state of the joint system 1 and 2 . The unnormalized state of the target system, given result $i$, is

$$
\begin{aligned}
\hat{\rho}_{i}^{3} & =\gamma_{i} \operatorname{tr}_{12}\left(\left(\tilde{\Pi}_{i}^{12} \otimes I^{3}\right)\left(\tilde{\rho}^{1} \otimes \sigma^{23}\right)\left(\tilde{\Pi}_{i}^{12} \otimes I^{3}\right)\right) \\
& =\gamma_{i} \operatorname{tr}_{12}\left(\left(\tilde{\Pi}_{i}^{12} \otimes I^{3}\right) U_{13}\left(\tilde{\sigma}^{12} \otimes \rho^{3}\right) U_{13}^{\dagger}\left(\tilde{\Pi}_{i}^{12} \otimes I^{3}\right)\right) \\
& =\left(\sqrt{\gamma_{i}}\left\langle\tilde{P}_{i}^{12}\left|U_{13}\right| \tilde{s}^{12}\right\rangle\right) \rho^{3}\left(\sqrt{\gamma_{i}}\left\langle\tilde{s}^{12}\left|U_{13}^{\dagger}\right| \tilde{P}_{i}^{12}\right\rangle\right) \\
& =A_{i}^{3} \rho^{3}\left(A_{i}^{3}\right)^{\dagger}
\end{aligned}
$$

where

$$
A_{i}^{3} \equiv \sqrt{\gamma_{i}}\left\langle\tilde{P}_{i}^{12}\left|U_{13}\right| \tilde{s}^{12}\right\rangle
$$

is an operator on system 3 alone. We have shown that if the joint system 2 and 3 is initially in a pure state and if the measurement on systems 1 and 2 is an ideal measurement described by one-dimensional projectors, then $\hat{\rho}_{i}^{3}$ is related to $\rho^{3}$ by an ideal quantum operation,

$$
\hat{\rho}_{i}^{3}=\mathcal{E}_{i}\left(\rho^{3}\right)=A_{i}^{3} \rho^{3}\left(A_{i}^{3}\right)^{\dagger} .
$$

We now notice that the probability for outcome $i$ is given by

$$
\operatorname{Pr}(i)=\gamma_{i} \operatorname{tr}\left(\left(\tilde{\rho}^{1} \otimes \sigma^{23}\right)\left(\tilde{\Pi}_{i}^{12} \otimes I^{3}\right)\right),
$$

which reduces to

$$
\operatorname{Pr}(i)=\operatorname{tr}\left(\hat{\rho}_{i}^{3}\right)=\operatorname{tr}\left(\rho^{3}\left(A_{i}^{3}\right)^{\dagger} A_{i}^{3}\right) .
$$

From the result characterizing unitarily reversible ideal quantum operations, it follows that Bob can achieve teleportation by doing a unitary operation on system 3 if and only if $\operatorname{Pr}(i)$ does not depend on the input state $\tilde{\rho}^{1}$ of system 1.

In the original paper on teleportation [2], it was noted incidentally that $\operatorname{Pr}(i)=\frac{1}{4}$, independent of the input state for system 1, for each of the four possible measurement results. We now see that this is in fact a sufficient condition to do teleportation in the scheme Bennett et al. were considering. In the original description of teleportation, the unitary operators on system 3 used to reconstruct the input state were given explicitly, and it was necessary to verify directly that these operators worked. An advantage of the present approach is that much less explicit computation has to be done in order to verify that teleportation is possible. Of course, one must construct the required unitary operators to perform teleportation. This is done by inverting the measurement operators $A_{i}^{3}$ found in the above construction.

To summarize, we have shown that the following are sufficient conditions to be able to perform teleportation:

1. Prepare the composite system so that the state of system 1 is unknown, but the state of the joint system 2 and 3 is known exactly, that is, is a pure state.

2. Perform a measurement on the joint system 1 and 2 that gives complete information about the posterior state of that system - that is, the joint system 1 and 2 is left in a pure state - but that gives no information about the prior state $\tilde{\rho}^{1}$ of system 1 .

Under these circumstances, given the result $i$ of the measurement, Bob can apply a unitary operation $U_{i}$ to system 3 , thereby putting it into the state $\rho^{3}$, the counterpart to the initial state $\tilde{\rho}^{1}$ of system 1 .

The teleportation scheme of Bennett et al. is an example of a scheme following this pattern. We can make some simple, yet powerful deductions about teleportation schemes of this type using the condition that the measurement probabilities $(\overline{6.10})$ be independent of $\rho$. This condition can be written as

$$
p_{i} / \gamma_{i}=\operatorname{tr}_{1}\left(\tilde{\rho}^{1} \operatorname{tr}_{23}\left(\left(I^{1} \otimes \sigma^{23}\right)\left(\tilde{\Pi}_{i}^{12} \otimes I^{3}\right)\right)\right),
$$


where $p_{i}$ is the constant value of the probability for result $i$. Since Eq. (6.12) holds for all input states $\tilde{\rho}^{1}$, we see that

$$
\begin{aligned}
\left(p_{i} / \gamma_{i}\right) I^{1} & =\operatorname{tr}_{23}\left(\left(I^{1} \otimes \sigma^{23}\right)\left(\tilde{\Pi}_{i}^{12} \otimes I^{3}\right)\right) \\
& =\left\langle s^{23}\left|\tilde{\Pi}_{i}^{12} \otimes I^{3}\right| s^{23}\right\rangle \\
& =\left\langle s^{23} \mid \tilde{P}_{i}^{12}\right\rangle\left\langle\tilde{P}_{i}^{12} \mid s^{23}\right\rangle
\end{aligned}
$$

where it is understood that $\left\langle s^{23} \mid \tilde{P}_{i}^{12}\right\rangle$ acts to the left as an operator on system 1 and to the right as an operator on system 3.

Consider the Schmidt decomposition [6] of the initial state $\left|s^{23}\right\rangle$ of the joint system 2 and 3 :

$$
\left|s^{23}\right\rangle=\sum_{j} \alpha_{j}\left|2_{j}\right\rangle\left|3_{j}\right\rangle
$$

Here the vectors $\left|2_{j}\right\rangle$ and $\left|3_{j}\right\rangle$ make up orthonormal bases for systems 2 and 3, respectively; we choose the phases so that the coefficients $\alpha_{j}$ are real and nonnegative. Now expand $\left|\tilde{P}_{i}^{12}\right\rangle$ as

$$
\left|\tilde{P}_{i}^{12}\right\rangle=\sum_{l m} \beta_{i, l m}\left|\overline{1}_{l}\right\rangle\left|2_{m}\right\rangle
$$

where the vectors $\left|\overline{1}_{l}\right\rangle$ make up any orthonormal basis for system 1. Combining the expansions of $\left|s^{23}\right\rangle$ and $\left|\tilde{P}_{i}^{12}\right\rangle$ gives

$$
\left\langle s^{23} \mid \tilde{P}_{i}^{12}\right\rangle=\sum_{j l} \alpha_{j} \beta_{i, l j}\left|\overline{1}_{l}\right\rangle\left\langle 3_{j}\right|
$$

Substituting this into (6.13), we see that the condition for teleportation becomes

$$
\left(p_{i} / \gamma_{i}\right) I^{1}=\sum_{j l l^{\prime}} \beta_{i, l j} \alpha_{j}^{2} \beta_{i, l^{\prime} j}^{*}\left|\overline{1}_{l}\right\rangle\left\langle\overline{1}_{l^{\prime}}\right| .
$$

An equivalent matrix expression is

$$
\mathbf{B}_{i} \mathbf{A}^{2} \mathbf{B}_{i}^{\dagger}=\left(p_{i} / \gamma_{i}\right) \mathbf{I}
$$

where $\mathbf{A}$ is the (positive) diagonal matrix with elements $A_{j k}=\alpha_{j} \delta_{j k}$ and $\mathbf{B}_{i}$ is the matrix with elements $B_{i, l m}=$ $\beta_{i, l m}$. Notice that the normalization of $\left|s^{23}\right\rangle$ can be written as $\operatorname{tr}\left(\mathbf{A}^{2}\right)=1$, and the normalization of $\left|\tilde{P}_{i}^{12}\right\rangle$ as $\operatorname{tr}\left(\mathbf{B}_{i}^{\dagger} \mathbf{B}_{i}\right)=1$.

Now write $\mathbf{B}_{i}$ in terms of a polar decomposition [6],

$$
\mathbf{B}_{i}=\mathbf{V}_{i} \mathbf{P}_{i}
$$

where $\mathbf{V}_{i}$ is a unitary matrix and $\mathbf{P}_{i}=\sqrt{\mathbf{B}_{i}^{\dagger} \mathbf{B}_{i}}$ is a positive matrix. Multiplying Eq. (6.18) on the left by $\mathbf{V}_{i}^{\dagger}$ and on the right by $\mathbf{V}_{i}$, we see that

$$
\mathbf{P}_{i} \mathbf{A}^{2} \mathbf{P}_{i}=\left(p_{i} / \gamma_{i}\right) \mathbf{I}
$$

Since by assumption $p_{i} / \gamma_{i}>0$, it follows that $\mathbf{A}$ and $\mathbf{P}_{i}$ both have nonzero determinants and thus are invertible. In particular, we have that $\alpha_{j}>0$ for all $j$. Furthermore, by manipulating Eq. (6.20), we can conclude that

$$
\mathbf{P}_{i}=\sqrt{p_{i} / \gamma_{i}} \mathbf{A}^{-1}
$$

Then the normalization condition for $\left|\tilde{P}_{i}^{12}\right\rangle$ implies that

$$
\frac{p_{i}}{\gamma_{i}}=\frac{\operatorname{tr}\left(\mathbf{P}_{i}^{2}\right)}{\operatorname{tr}\left(\mathbf{A}^{-2}\right)}=\frac{1}{\operatorname{tr}\left(\mathbf{A}^{-2}\right)} \equiv k
$$

where $k$ is a constant independent of the measurement result $i$. Define now, for each $i$, a new orthonormal basis for system 1 by

$$
\left|1_{i, j}\right\rangle \equiv \sum_{l} V_{i, l j}\left|\overline{1}_{l}\right\rangle
$$

in terms of which the expansion of $\left|\tilde{P}_{i}^{12}\right\rangle$ becomes a Schmidt decomposition,

$$
\left|\tilde{P}_{i}^{12}\right\rangle=\sum_{j m} P_{i, j m}\left|1_{i, j}\right\rangle\left|2_{m}\right\rangle=\sqrt{k} \sum_{j} \alpha_{j}^{-1}\left|1_{i, j}\right\rangle\left|2_{j}\right\rangle,
$$

with the system 2 basis in the Schmidt decomposition the same as the system 2 basis in the Schmidt decomposition of $\left|s^{23}\right\rangle$.

The last ingredient comes from the completeness relation 6.5$)$ :

$$
\begin{aligned}
I^{1} & =\left\langle 2_{j}\left|I^{12}\right| 2_{j}\right\rangle \\
& =\sum_{i} \gamma_{i}\left\langle 2_{j} \mid \tilde{P}_{i}^{12}\right\rangle\left\langle\tilde{P}_{i}^{12} \mid 2_{j}\right\rangle \\
& =\frac{1}{\alpha_{j}^{2}} \sum_{i} p_{i}\left|1_{i, j}\right\rangle\left\langle 1_{i, j}\right| .
\end{aligned}
$$

Taking the trace of both sides gives $\alpha_{j}=1 / \sqrt{d}$ for all $j$, which implies that $k=1 / d^{2}$ and

$$
p_{i}=\frac{\gamma_{i}}{d^{2}}
$$

Thus we find that the initial state vector of the joint system 2 and 3 ,

$$
\left|s^{23}\right\rangle=\frac{1}{\sqrt{d}} \sum_{j}\left|2_{j}\right\rangle\left|3_{j}\right\rangle
$$

is maximally entangled, and the measurement state vectors,

$$
\left|\tilde{P}_{i}^{12}\right\rangle=\frac{1}{\sqrt{d}} \sum_{j}\left|1_{i, j}\right\rangle\left|2_{j}\right\rangle,
$$

are maximally entangled states of the joint system 1 and 2 . 
What we have shown is a complete characterization of teleportation schemes of the type introduced in [2]. Any maximally entangled state of the joint system 2 and 3 can be used as the initial state of 2 and 3, and any set of maximally entangled states of the joint system 1 and 2 which satisfy the completeness relation (6.5) can be used to define the measurement operators on 1 and 2. For a maximally entangled state, one of the orthonormal bases in the Schmidt decomposition can be chosen arbitrarily, so it is always possible to put all these maximally entangled states in the canonical form of Eqs. 6.27) and (6.28), in which all the Schmidt decompositions share a common basis in system 2. These conditions are both necessary and sufficient to do teleportation provided it is assumed that the state of systems 2 and 3 is pure, and the POVM elements measured by Alice are one-dimensional. It is clear that the teleportation scheme introduced in [2] satisfies these conditions.

\section{CONCLUSION}

We have proved a general result characterizing unitarily reversible ideal quantum operations. In the context of quantum measurements this result has an intuitive physical meaning: an ideal quantum measurement is unitarily reversible if and only if no information about the prior quantum state is obtained as a result of the measurement. The characterization has two limitations to be addressed by further work: it needs to be extended to apply to any quantum operation, not just ideal ones, and it should allow the reversal to be performed nonunitarily, provided the reversal is still deterministic.

We have shown how quantum teleportation can be understood in terms of the general problem of reversing quantum operations, thereby demonstrating the crucial connection between teleportation and the fact that no information about the state to be teleported is gained during the process. We have used the condition for unitarily reversing an ideal quantum operation to characterize completely teleportation schemes of the type introduced by Bennett et al.

\section{ACKNOWLEDGMENTS}

We thank H. Barnum, C. A. Fuchs, and B. Schumacher for instructive and enjoyable discussions about quantum information. We thank C. A. Fuchs, in particular, for suggesting to us a connection between reversing measurements and teleportation. This work was supported in part by the Office of Naval Research (Grant No. N00014-93-1-0116) and the Phillips Laboratory (Grant No. F29601-95-0209). We thank the Institute for Theoretical Physics for its hospitality and for the support of the National Science Foundation (Grant No. PHY9407194). MN acknowledges financial support from the Australian-American Educational Foundation (Fulbright Commission).

[1] H. Mabuchi and P. Zoller, Phys. Rev. Lett. 76, 3108 (1996).

[2] C. H. Bennett et al., Phys. Rev. Lett. 70, 1895 (1993).

[3] K. Kraus, States, Effects, and Operations (SpringerVerlag, Berlin, 1983).

[4] B. Schumacher, LANL e-print quant-ph/9604023, to appear in Phys. Rev. A (unpublished).

[5] C. W. Gardiner, Quantum Noise (Springer-Verlag, Berlin, 1991).

[6] A. Peres, Quantum Theory: Concepts and Methods (Kluwer Academic, Dordrecht, 1993).

[7] S. L. Braunstein, A. Mann, and M. Revzen, Phys. Rev. Lett. 68, 3259 (1992).

[8] P. W. Shor, Phys. Rev. A 52, 2493 (1995).

[9] A. Ekert and C. Macchiavello, LANL e-print quantph/9602022 (unpublished).

[10] E. Knill and R. Laflamme, LANL e-print quantph/9604034 (unpublished).

[11] M. A. Nielsen, LANL e-print quant-ph/9606012 (unpublished).

[12] B. Schumacher and M. A. Nielsen, to appear in Phys. Rev. A (unpublished).

[13] P. W. Shor, LANL e-print quant-ph/9605011 (unpublished).

[14] A. M. Steane, LANL e-print quant-ph/9605021 (unpublished).

[15] It is trivial to generalize the discussion of Sec. $\mathrm{VI}$ to measurement operators of the form

$$
\sqrt{\gamma_{i}}\left(\tilde{V}_{i} \tilde{\Pi}_{i}^{12}\right) \otimes I^{3}=\sqrt{\gamma_{i}}\left|\tilde{Q}_{i}^{12}\right\rangle\left\langle\tilde{P}_{i}^{12}\right| \otimes I^{3},
$$

where $\tilde{V}_{i}$ is any unitary operator on the joint system 1 and 2 and $\left|\tilde{Q}_{i}^{12}\right\rangle \equiv \tilde{V}_{i}\left|\tilde{P}_{i}^{12}\right\rangle$. To keep the clutter of the notation at its present level, we stick to the simpler case in Sec. $\mathrm{VI}$. 